\title{
Interferon- $\alpha$ Treatment for Growing Teratoma Syndrome of the Testis
}

\author{
Masahiro Inoue Shin-ichi Hisasue Mika Nagae Toshiyuki China \\ Keisuke Saito Shuji Isotani Raizo Yamaguchi Hisamitsu Ide \\ Satoru Muto Shigeo Horie
}

Department of Urology, Teikyo University School of Medicine, Tokyo, Japan

\section{Key Words}

Testicular tumor $\cdot$ Immature teratoma $\cdot$ Growing teratoma syndrome $\cdot$ Interferon- $\alpha$

\begin{abstract}
A 23-year-old man with a right scrotal mass and back pain was referred for further treatment after right radical orchiectomy for testicular cancer. CT scans brought by the patient showed extensive metastasis to the retroperitoneal lymph nodes with no lung involvement. $\alpha$ Fetoprotein and human chorionic gonadotropin were elevated preoperatively $(384 \mathrm{ng} / \mathrm{ml}$ and $112 \mathrm{mIU} / \mathrm{ml}$, respectively). Confirmation of the histopathologic examination revealed a mixed germ cell tumor (95\% immature teratoma and 5\% embryonal carcinoma). We started the patient on chemotherapy with bleomycin, etoposide, and cisplatin (BEP). After a single course, tumor markers began to normalize, but there was radiologic evidence of continued growth of the retroperitoneal mass and new metastases in the lung. The patient was given 2 courses of salvage chemotherapy with etoposide, ifosfamide, and cisplatin (VIP). However, the mass and lung metastases continued to progress, and the patient was growing rapidly intolerant of the side effects of treatment (i.e., nausea, appetite loss, and pancytopenia). After thorough discussion with the patient and his family, we decided to start the patient on interferon (IFN)- $\alpha$ therapy. Natural, nonrecombinant IFN- $\alpha$ (OIF, Otsuka, Japan) 5,000,000 IU was administered twice weekly with approval of the ethics committee of our institution. The patient responded moderately with marked deceleration of tumor growth and stabilization of the lung metastases. He is alive and well at 16 months on IFN- $\alpha$ therapy.
\end{abstract}

\section{Introduction}

Growing teratoma syndrome (GTS) is a rare entity characterized by accelerated growth of masses in the retroperitoneum or other locations during or after systemic chemotherapy 
for treatment of a nonseminomatous germ cell tumor (NSGCT). Three criteria define this syndrome: enlarging metastatic masses, normalized serum markers, and no viable cancer cells in the teratoma. We report a case of GTS which was treated with interferon (IFN)- $\alpha$ leading to long-term survival.

\section{Case Report}

A 23-year-old man with a right scrotal mass and back pain was referred for further treatment after a right radical orchiectomy for testicular cancer. Preoperative CT scans revealed extensive metastasis to the retroperitoneal lymph nodes without lung metastasis. $\alpha$-Fetoprotein (AFP) and human chorionic gonadotropin (hCG) were elevated preoperatively (384 $\mathrm{ng} / \mathrm{ml}$ and $112 \mathrm{mIU} / \mathrm{ml}$, respectively). The patient had a right radical orchiectomy under the clinical diagnosis of stage IIB testicular cancer. The histopathologic examination revealed a mixed germ cell tumor consisting of $95 \%$ immature teratoma and $5 \%$ embryonal carcinoma. According to the International Germ Cell Consensus Classification (IGCCC), the patient's prognosis was 'good'. One week after surgery, the AFP and hCG levels began to normalize (310 ng/ml and $16.8 \mathrm{mIU} / \mathrm{ml}$, respectively).

The patient was referred to our institution 1 month after surgery, with new radiologic evidence of an enlarging retroperitoneal mass and multiple lung metastases. The AFP level was slightly higher $(548 \mathrm{ng} / \mathrm{ml})$, but the hCG level had continued to normalize $(9.4 \mathrm{mIU} / \mathrm{ml})$. Abdominal CT showed a huge mass surrounding the aorta and inferior vena cava $(11 \times 6 \times$ $16 \mathrm{~cm}$; fig. 1a), which was determined to be metastatic spread to the retroperitoneal lymph nodes. Lung CT ( $5 \mathrm{~mm}$ sliced) showed 38 new lesions in the lung, also evidencing metastatic spread of cancer. The largest lesion was $2.0 \mathrm{~cm}$ in diameter (fig. 1b).

We immediately started the patient on combined chemotherapy with bleomycin, etoposide, and cisplatin (BEP). By day 20, both tumor markers had decreased in value (AFP 274 $\mathrm{ng} / \mathrm{ml}$ and $\mathrm{hCG} 1.6 \mathrm{mIU} / \mathrm{ml}$ ). Despite this excellent response, there was a rapid increase in the number of lung lesions (from 38 to 72 ) (fig. 1c, d; fig. 2). The patient was given 2 courses of salvage chemotherapy with etoposide, ifosfamide, and cisplatin (VIP). After salvage therapy, the tumor markers were markedly decreased (AFP $28.4 \mathrm{ng} / \mathrm{ml}$ and hCG $<0.1$ $\mathrm{mIU} / \mathrm{ml}$ ), but the retroperitoneal mass (fig. 1e) and lung metastases (fig. 1f) continued to grow, with lung lesions now numbering 105. Moreover, the adverse effects of treatment, including nausea, appetite loss, and pancytopenia, were growing increasingly intolerable to the patient. After a long discussion with the patient and his family, we decided to stop VIP treatment and start the patient on natural, nonrecombinant IFN- $\alpha$. IFN- $\alpha$ (OIF, Otsuka, Japan) 5,000,000 IU was administered twice weekly, after approval was obtained from the ethics committee of our institution.

IFN- $\alpha$ administration resulted in a marked decrease in the rate of progression of the retroperitoneal mass (tumor doubling time was about 2 months before IFN- $\alpha$ and about 12 months after IFN- $\alpha$; fig. 1g), and the lung metastases stabilized, with no new lesions detected (fig. 1h). Just before IFN- $\alpha$ treatment, the tumor markers were normalized (AFP $3.4 \mathrm{ng} / \mathrm{ml}$ and $\mathrm{hCG}<0.1 \mathrm{mIU} / \mathrm{ml}$ ). The patient continues to receive IFN- $\alpha$ and is alive 16 months after IFN- $\alpha$ treatment was started. 


\section{Discussion}

We report here a rare case of a patient with GTS who responded well to treatment with IFN- $\alpha$. The tumor, arising after chemotherapy for an NSGCT, was massive in size and extent, exhibited a high rate of growth, and could not be resected. The case is remarkable for its strong response to IFN- $\alpha$, which resulted in stabilization of the disease and long-term survival.

GTS was first highlighted by Logothetis in 1982 [1]. It is described as an enlarging mature teratoma that appears during or after chemotherapy for an NSGCT, accompanied by normal serum levels of AFP and hCG. Three criteria define this syndrome: enlarging metastatic masses, normalized serum markers, and no viable cancer cells in the mature teratoma [1]. GTS is rare, occurring in only $2-7 \%$ of NSGCT [2]. The preferred approach to treatment is complete surgical resection, since teratomas are known to be highly resistant to chemotherapy and radiation therapy. Andre et al. [3] reported the outcome of surgical resection in 30 patients with GTS. Twenty-four patients underwent total resection, and 6 patients were treated with partial resection. The recurrence rate was markedly disparate between the two groups, with a rate of $4 \%$ in the total resection group and $83 \%$ in the partial resection group. Hence, good results are often achieved with surgery alone, provided a complete resection can be performed.

With inoperable GTS, however, there is no established treatment, and the clinical outcome is generally poor. Rustin et al. [4] reported their experience with IFN therapy in 10 cases of chemotherapy-resistant, inoperable teratoma. Only 2 of the 10 cases achieved stabilization of disease. Although the exact mechanism of the efficacy of IFN- $\alpha$ is still unknown, the treatment is considered safe, and the tolerability is high. Certainly, it warrants consideration in cases of inoperable GTS, when other options have been exhausted.

In addition to IFN- $\alpha$, some attempts have been made to treat inoperable GTS with molecular-targeted therapies. Bevacizumab, a humanized monoclonal antibody that recognizes and blocks vascular endothelial growth factor A, was applied to a case of inoperable GTS, but resulted in disease progression [5]. Vaughn et al. [6] had better results in 3 patients with GTS treated with CDK4/6 inhibitor, which produced a partial response in 1 and stabilization of disease in the other 2 . This drug is promising and we hope that it will be available in the near future for patients with GTS.

In this case, we could not carry out a biopsy for the tumor before IFN- $\alpha$ treatment. The tumor progression was extremely rapid, especially in lung, and life-threatening. The pathological diagnosis for testicular cancer as a primary site was immature teratoma in over $90 \%$ of the specimens and the metastases were resistant to chemotherapy. Therefore, we believed that metastatic sites are immature teratoma. However, a biopsy for the tumor is necessary to confirm the exact relationship between GTS and the treatment response. When we have to move on to another treatment for this case, we should consider a biopsy again in advance.

We report here an extremely rare case of GTS which was responsive to treatment with IFN- $\alpha$. The treatment contributed not only to tumor stabilization but also to long-term survival. The patient is alive and well 16 months after treatment was started. IFN- $\alpha$ has been established to be safe and well tolerated by patients, and should not be overlooked in difficult cases of inoperable GTS. 
Inoue et al.: Interferon- $\alpha$ Treatment for Growing Teratoma Syndrome of the Testis

\section{References}

1 Logothetis CJ, Samuels ML, Trindade A, Johnson DE: The growing teratoma syndrome. Cancer 1982;50:1629-1635.

-2 Jeffery GM, Theaker JM, Lee AH, Blaquiere RM, Smart CJ, Mead GM: The growing teratoma syndrome. Br J Urol 1991;67:195-202.

-3 Andre F, Fizazi K, Culine S, Droz J, Taupin P, Lhomme C, et al: The growing teratoma syndrome: results of therapy and long-term follow-up of 33 patients. Eur J Cancer 2000;36:1389-1394.

4 Rustin GJ, Kaye SB, Williams CJ, Newlands ES, Bagshawe KD, Toy JL: Response of differentiated but not anaplastic teratoma to interferon. Br J Cancer 1984;50:611-616.

$\checkmark 5$ Mego M, Reckova M, Sycova-Mila Z, Obertova J, Brozmanova K, Salek T, et al: Bevacizumab in a growing teratoma syndrome. Case report. Ann Oncol 2007;18:962-963.

6 Vaughn DJ, Flaherty K, Lal P, Gallagher M, O’Dwyer P, Wilner K, et al: Treatment of growing teratoma syndrome. N Engl J Med 2009;360:423-424. 

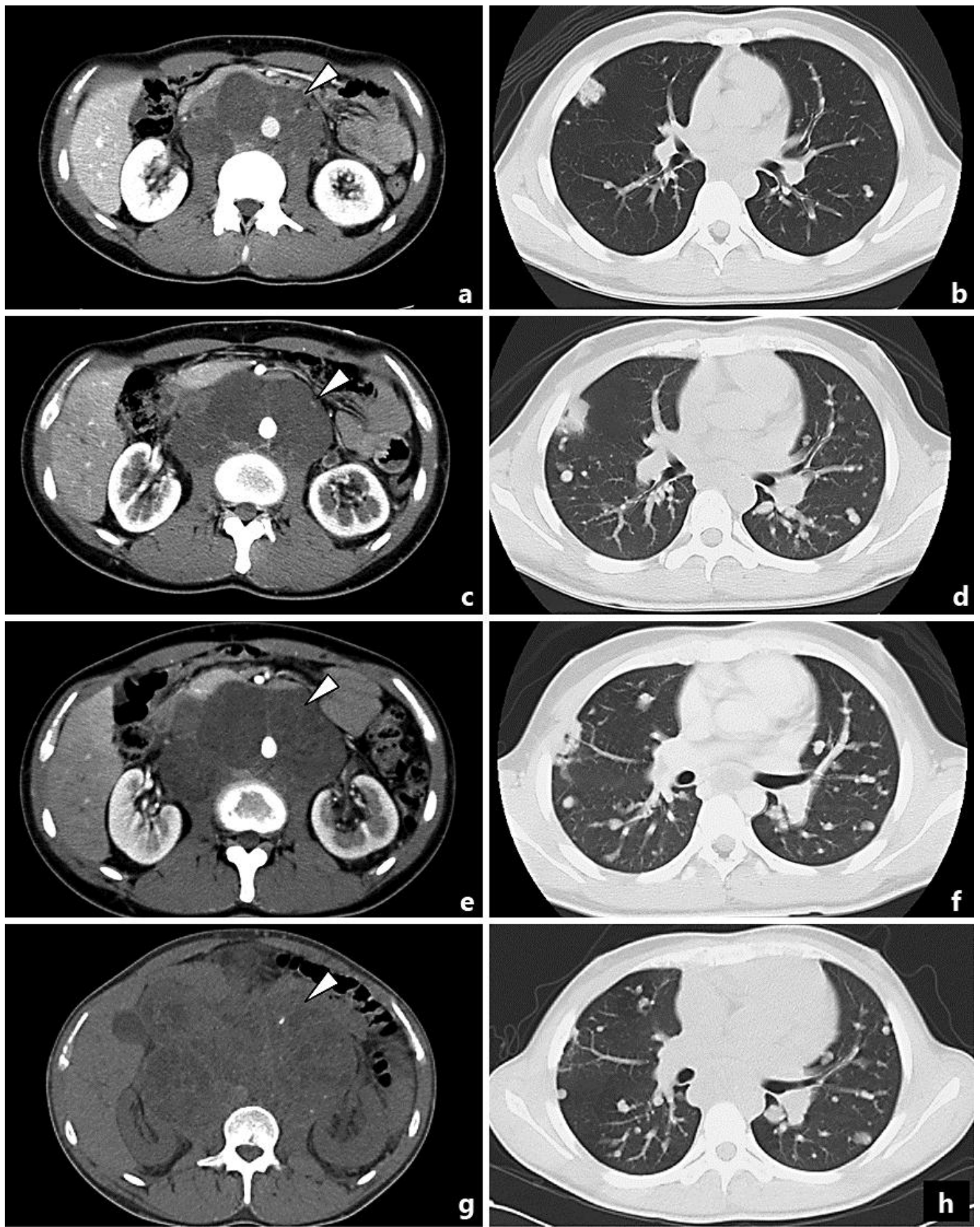

Fig. 1. Abdominal CT (a) and lung CT (b) at the patient's presentation. Abdominal CT shows a huge retroperitoneal lymph node metastasis surrounding the vena cava and aorta. Lung CT shows multiple lung metastases. Abdominal CT (c) and lung CT (d) after 1 course of BEP therapy. The retroperitoneal mass and lung metastases were progressive. Abdominal CT (e) and lung CT (f) after 2 courses of VIP therapy. The retroperitoneal mass and lung metastases were still progressive. Abdominal CT (g) and lung CT (h) 1 year after IFN- $\alpha$. The retroperitoneal mass is still growing slowly; however, the progression of the lung metastases is stabilized. The arrowhead shows the retroperitoneal lymph node metastasis. 


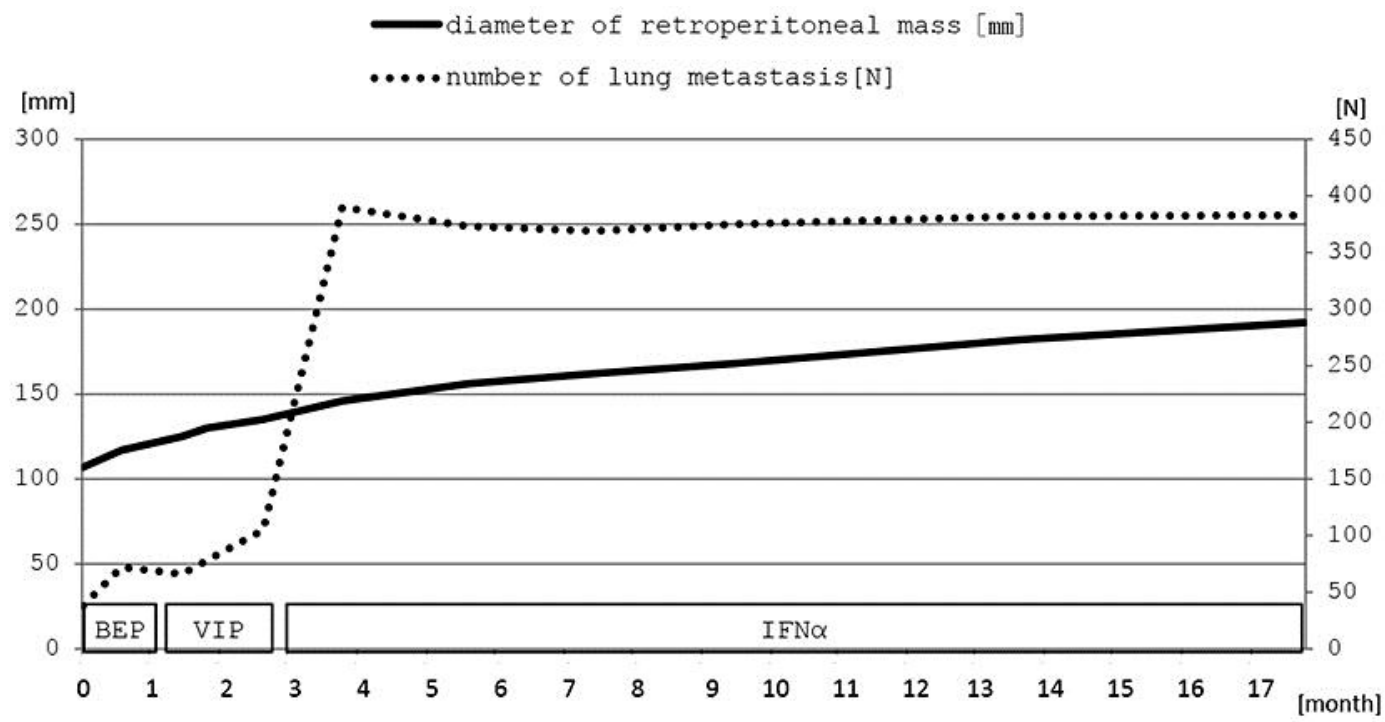

Fig. 2. Summary of retroperitoneal metastatic tumor size and number of lung metastases during various courses of treatment. 